\title{
Numerical study of the stability of some explicit finite-difference methods for oscillatory advection
}

\author{
John Noye* David McInerney ${ }^{\dagger}$ \\ (Received 7 August 2000)
}

\begin{abstract}
The basis for modelling unsteady transport in fluids is the onedimensional advection equation. When high-order finite-difference methods for constant velocity advection are applied to the variable

*National Tidal Facility, Flinders University of South Australia. Seconded from the Department of Applied Mathematics, University of Adelaide, Australia.

${ }^{\dagger}$ Department of Applied Mathematics, University of Adelaide, Australia.

${ }^{0}$ See http: //anziamj . austms .org. au/V42/CTAC99/Noye for this article and ancillary services, (c) Austral. Mathematical Soc. 2000. Published 27 Nov 2000.
\end{abstract}


velocity case, they generally degenerate at least one order. For example, Crowley's formula, which is second order for constant velocity advection, is only first order when used with variable velocities. Modification of Crowley's formula has recently yielded three formulae which are second order in the variable velocity case. Two commonly used local stability analyses have been carried out on these formulae with conflicting results. The resulting stability regions have been studied by means of a series of numerical experiments using an oscillatory advection velocity to determine their applicability in tidal streams.

\section{Contents}

1 Introduction

$\mathrm{C} 1078$

2 Mathematical Formulation

C1080

3 Numerical Stability

C1083

3.1 First Stability Criterion: locally constant velocity . . . . . C1083

3.2 Second Stability Criterion: "frozen coefficients" . . . . . . C1084

4 Numerical Tests

C1085

4.1 Test Problem 1: Velocity periodic in time. . . . . . . . . . C1085

4.2 Test Problem 2: Spatial periodicity of $2 \Delta x \ldots$. . . . . . C1091

5 Conclusion

C1094 


\section{Introduction}

The accurate modelling of advective terms in the advection-diffusion equation which governs the spread of contaminants in a fluid, has been the subject of ongoing research for some time and a number of accurate high-order finitedifference solutions for the advection equation with constant coefficients have been developed (see, for example, Leith [3] and Noye [6, 7]).

When a high-order finite-difference method for constant velocity advection is applied in the variable velocity situation, it generally degenerates at least one order of convergence. For example, Crowley's second-order formula for the constant coefficient advection equation (Leith [3] and Crowley [1]) reduces to first order when used in the variable velocity case [8].

Noye [8] outlined a technique which can modify a finite-difference formula for approximately solving the one-dimensional advection equation with a source term, enabling it to retain its constant coefficient order in the variable coefficient case. The method was illustrated by application to Crowley's formula. The three resulting finite-difference equations (FDE's) use only grid values of the time and space varying velocity and source, which are generally 
all that are available in practical situations.

In this paper the two main criteria applied to variable coefficient FDE's to ensure stability, are tested numerically. Both techniques use a form of local von Neumann stability analysis [9] of the difference equations. The first method uses the related constant coefficient difference equation to give stability conditions for the variable coefficient case [10]. The second technique involves the use of "frozen" coefficients in the variable coefficient FDE to give the stability criteria [4]. In both cases the finite-difference method is assumed to be numerically stable if the resulting stability condition is satisfied at every point in the solution domain.

These two criteria have been applied to the three FDE's developed by Noye [8], and the resulting stability range determined by means of numerical tests in which the velocity and the solution of the problem oscillate in time and space. This choice was made because of its practical application to the spread of pollutants and of pelagic larvae in oscillatory tidal streams.

The first of these techniques yields the criteria

$$
|c|_{\max } \leq 1
$$

for all three formulae, where $c$ is the Courant number given by $c=u \Delta t / \Delta x$. In all cases, numerical results from the test problems indicate they are stable so long as (1) is satisfied. However, many test runs were also stable for $|c|_{\max }>1$, up to 1.5 , so long as the spatial grid size was not too small.

The second technique yields more restrictive criteria than (1), criteria 
which are often not satisfied by the formulae even when (1) is satisfied. Yet the FDE's remained stable up to ten million time steps when $|c|_{\max }=1.1$.

\section{Mathematical Formulation}

Consider the simulation of advection of a smooth scalar quantity $\hat{\tau}(x, t)$ in a moving fluid with a smooth space and time varying velocity field $u(x, t)$, by

$$
\frac{\partial \hat{\tau}}{\partial t}+u(x, t) \frac{\partial \hat{\tau}}{\partial x}=F(x, t), \quad 0 \leq x \leq 1, \quad 0<t \leq T,
$$

where $x$ represents a space coordinate, $t$ the time variable, $T$ the final time of interest and $F(x, t)$ is a smooth source function. To solve this equation numerically, an initial condition $\hat{\tau}(x, 0)$ is prescribed on $0 \leq x \leq 1$, as well as a set of boundary conditions is specified by $\hat{\tau}(0, t)$ and $\hat{\tau}(1, t)$ for $0<t \leq T$.

Finite-difference approximations $\tau_{j}^{n}$ for the values of $\hat{\tau}\left(x_{j}, t_{n}\right)$, at interior points $x_{j}=j \Delta x, j=1(1) J-1$ and time $t_{n}=n \Delta t, n=1(1) N, \Delta x=1 / J$ and $\Delta t=T / N$, may be computed using FDE's so long as they are consistent with (2) and stable with respect to the propagation of round-off error (Lax and Richtmyer [2]). The notation $p(q) r$ represents the set of integers $p, p+q$, $p+2 q, \ldots$, not exceeding the integer $r$.

One approach to develop a FDE to approximately solve (2), based on Crowley's second-order explicit FDE for the constant coefficient advection 
equation, is to simply add $\Delta t F_{j}^{n}$ to the evaluation of $\tau_{j}^{n+1}$, to incorporate the effect of the source term, where $F_{j}^{n}=F\left(x_{j}, t_{n}\right)$. Also the constant Courant number $c$ is replaced by $c_{j}^{n}=u\left(x_{j}, t_{n}\right) \Delta t / \Delta x$, giving

$$
\tau_{j}^{n+1}=\frac{1}{2}[c(c+1)]_{j}^{n} \tau_{j-1}^{n}+\left[1-c^{2}\right]_{j}^{n} \tau_{j}^{n}+\frac{1}{2}[c(c-1)]_{j}^{n} \tau_{j+1}^{n}+\Delta t F_{j}^{n} .
$$

An upwind (UW) modified second-order form of equation (3) for approximately solving (2) is (Noye [8])

$$
\begin{aligned}
\tau_{j}^{n+1}= & \frac{1}{2}\left[c+c^{2}+d+|d|\right]_{j}^{n} \tau_{j-1}^{n}+\left[1-c^{2}-|d|\right]_{j}^{n} \tau_{j}^{n} \\
& -\frac{1}{2}\left[c-c^{2}+d-|d|\right]_{j}^{n} \tau_{j+1}^{n}+\Delta t[F+G]_{j}^{n} .
\end{aligned}
$$

in which

$$
d=\frac{(\Delta t)^{2}}{2 \Delta x}\left(\frac{\partial u}{\partial t}-u \frac{\partial u}{\partial x}\right), G=\frac{\Delta t}{2}\left(\frac{\partial F}{\partial t}-u \frac{\partial F}{\partial x}\right) .
$$

A centred space (CS) modified Crowley type second-order FDE for approximately solving (2) is (Noye [8])

$$
\tau_{j}^{n+1}=\frac{1}{2}\left[c+c^{2}+d\right]_{j}^{n} \tau_{j-1}^{n}+\left[1-c^{2}\right]_{j}^{n} \tau_{j}^{n}-\frac{1}{2}\left[c-c^{2}+d\right]_{j}^{n} \tau_{j+1}^{n}+\Delta t[F+G]_{j}^{n}
$$

A semi-Lagrangian based second-order finite-difference formula for approximately solving (2) is (see [8])

$$
\tau_{j}^{n+1}=\frac{[h(h+1)]_{j}^{n}}{2} \tau_{j-1}^{n}+\left[1-h^{2}\right]_{j}^{n} \tau_{j}^{n}+\frac{[h(h-1)]_{j}^{n}}{2} \tau_{j+1}^{n}+\Delta t[F+G]_{j}^{n},
$$


which is the same as (3) with $h=c+d$ replacing $c$, and $F+G$ replacing $F$.

In general only discrete velocity and source fields are available; that is values of $u$ and $F$ are known only at grid points $\left(x_{j}, t_{n}\right)$ in the solution domain. In this case an approximate value of $d_{j}^{n}$ must be computed to second order from the given values of $u_{j}^{n}$. This may be achieved by using grid values of $u$ to approximate $\partial u /\left.\partial t\right|_{j} ^{n}$ and $\partial u /\left.\partial x\right|_{j} ^{n}$ to first order.

Use of two-point forward-time and centred-space approximations gives

$$
d_{j}^{n} \simeq \frac{1}{4}\left\{2\left(c_{j}^{n+1}-c_{j}^{n}\right)-c_{j}^{n}\left(c_{j+1}^{n}-c_{j-1}^{n}\right)\right\} .
$$

This leads to

$$
h_{j}^{n} \simeq \frac{1}{4}\left\{2\left(c_{j}^{n+1}+c_{j}^{n}\right)-c_{j}^{n}\left(c_{j+1}^{n}-c_{j-1}^{n}\right)\right\}
$$

for use in (6). In addition an approximate value of $G_{j}^{n}$ computed to at least second order from the given grid values of $F$ may be found by differencing $\partial F /\left.\partial t\right|_{j} ^{n}$ and $\partial F /\left.\partial x\right|_{j} ^{n}$ to first order in a similar manner to the differencing of the derivatives of $u$ in $d_{j}^{n}$, yielding

$$
G_{j}^{n} \simeq \frac{1}{4}\left\{2\left(F_{j}^{n+1}-F_{j}^{n}\right)-c_{j}^{n}\left(F_{j+1}^{n}-F_{j-1}^{n}\right)\right\}
$$




\section{Numerical Stability}

Before these FDE's are applied, the range of values of $c, d$ and $h$ for which (4), (5) and (6) are numerically stable [9] should be established. Since the equations involved are linear, the stability of their homogeneous parts (obtained by setting $F=G=0$ ) is all that is required. The resulting homogeneous variable coefficient FDE is usually examined for stability in one of two ways, both using the concept of 'local' stability.

\subsection{First Stability Criterion: locally constant velocity}

Assuming $u$ is locally constant in the FDE applied at the grid point $\left(x_{j}, t_{n}\right)$, so that $c_{j}^{n+1}=c_{j-1}^{n}=c_{j+1}^{n}=c_{j}^{n}=c$, a constant, and $d_{j}^{n}=0$, a constant coefficient scheme is obtained "that can be used to give stability conditions for the same scheme applied to equations with variable coefficients" (Strikwerda [10]). For equations (4), (5) and (6),

$$
\tau_{j}^{n+1}=\frac{1}{2} c(c+1) \tau_{j-1}^{n}+(1-c)(1+c) \tau_{j}^{n}+\frac{1}{2} c(c-1) \tau_{j+1}^{n}
$$

is obtained. This is Crowley's equation [1] for the approximate solution of the constant coefficient advection equation. Since (8) is von Neumann stable for $|c| \leq 1$ [3], Strikwerda's condition implies that the formulae (4), (5) and (6) will be stable so long as

$$
\left|c_{j}^{n}\right| \leq 1 \text { for all } j \text { and } n .
$$




\section{Numerical Stability}

This requires

$$
|c|_{\max }=\max _{j, n}\left|c_{j}^{n}\right| \leq 1
$$

if (9) is to be satisfied at every grid point in the computational domain.

\subsection{Second Stability Criterion: "frozen coefficients"}

A von Neumann stability analysis can be applied locally to a variable coefficient FDE [4] if the coefficients of the FDE are "frozen" to a fixed value in the computational molecule. If the resulting stability condition is satisfied at every interior grid point the method is then assumed stable.

Instability is essentially a local phenomenon due to the high frequency modes being the most unstable [5]. Therefore, for the computational molecule centred on $\left(x_{j}, t_{n}\right)$, we take $c_{j}^{n}$ to be "frozen" at the value $c$, and $d_{j}^{n}$ to be frozen at the value $d$. A von Neumann analysis of the homogeneous FDE which is then obtained from (4), namely

$$
\begin{gathered}
\tau_{j}^{n+1}=\frac{1}{2}\left(c+c^{2}+d+|d|\right) \tau_{j-1}^{n}+\left(1-c^{2}-|d|\right) \tau_{j}^{n} \\
-\frac{1}{2}\left(c-c^{2}+d-|d|\right) \tau_{j+1}^{n},
\end{gathered}
$$

which yields the following stability criterion (see [8]):

$$
\begin{aligned}
& \text { if } d<0, \quad d \geq-1-2 c \quad \text { and } \quad d \geq c^{2}-1, \\
& \text { if } d>0, \quad d \leq 1-2 c \quad \text { and } \quad d \leq 1-c^{2},
\end{aligned}
$$


at each grid point. This region of the $(c, d)$ plane is shown in Figure 1(a).

Similarly a stability analysis of the reduced form of (5), namely,

$$
\tau_{j}^{n+1}=\frac{1}{2}\left(c+c^{2}+d\right) \tau_{j-1}^{n}+\left(1-c^{2}\right) \tau_{j}^{n}-\frac{1}{2}\left(c-c^{2}+d\right) \tau_{j+1}^{n},
$$

leads to the criterion (see Appendix A) to be satisfied at each grid point:

$$
\begin{aligned}
|c| \leq 1 \quad \text { and } \quad-2 c \leq d<0 & \text { if } \quad c>0 \\
0<d \leq-2 c & \text { if } c<0 .
\end{aligned}
$$

The corresponding region of the $(c, d)$ plane is shown in Figure 1(b).

Finally when (6) is considered, application of a von Neumann analysis with $h_{j}^{n}$ taken to be constant leads to the requirement that $|h|=|c+d| \leq 1$, at each grid point if stability is to be guaranteed. This leads to

$$
-1-c \leq d \leq 1-c,
$$

which is the region shown in Figure 1(c).

\section{Numerical Tests}

\subsection{Test Problem 1: Velocity periodic in time}

The $1 \mathrm{D}$ variable velocity advection equation with source term (2) has been solved on the computational domain $0 \leq x \leq 1,0<t \leq T$ using the standard 


\section{$4 \quad$ Numerical Tests}
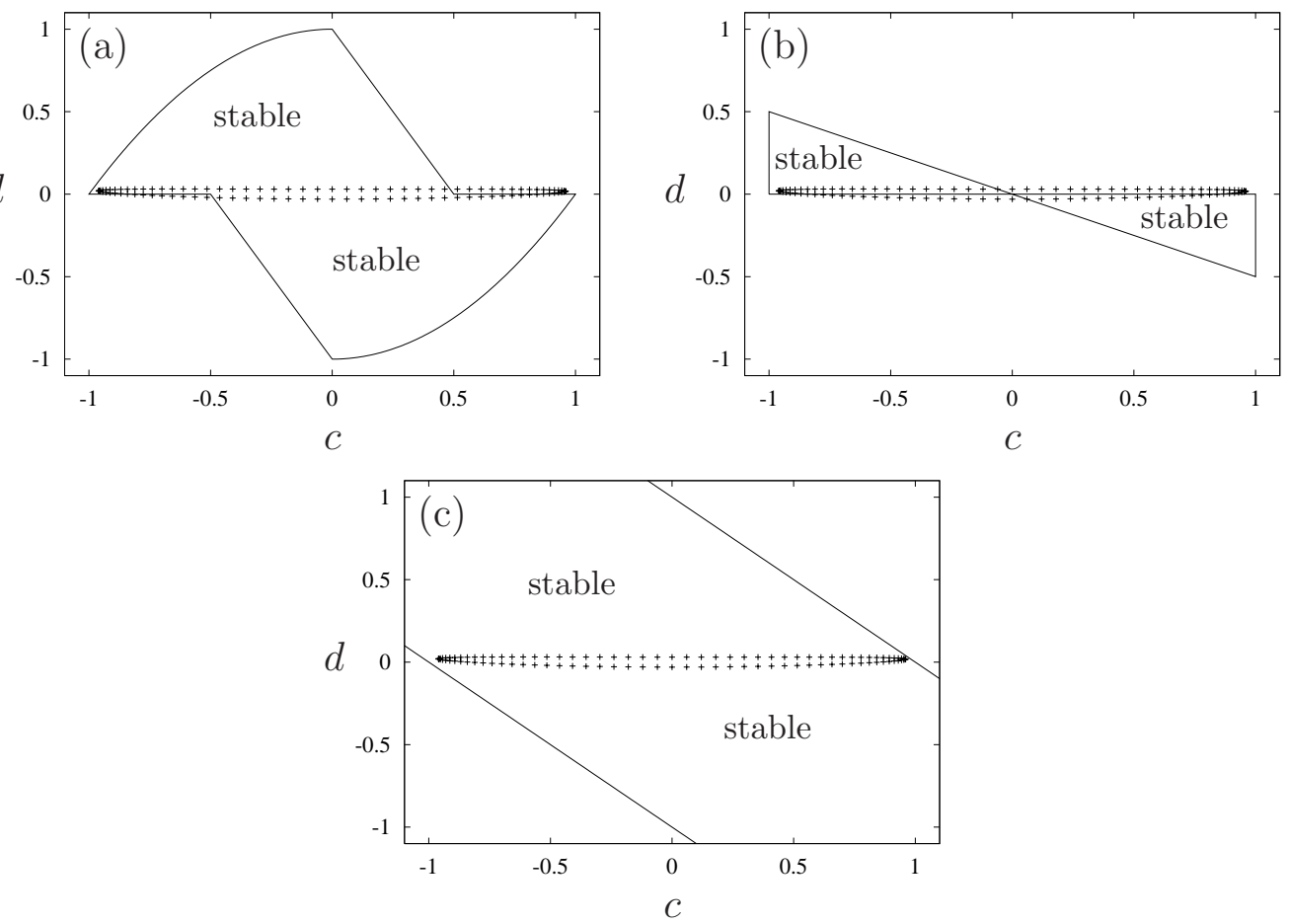

Figure 1: von Neumann stability region in $(c, d)$ space of (a) upwind modification, (b) centred space modification, (c) semi-Lagrangian version, of Crowley's formula. The values of $(c, d)$ used over one period of both test problems, when $|c|_{\max }=1.0, J=100$ at the point $x=0.01$, are indicated + . 
FDE (3) and the new FDE's (4), (5) and (6). The non-negative solution

$$
\hat{\tau}(x, t)=\exp \left\{-50(2 x-1+\sin \{2 \pi t\})^{2}\right\}
$$

of equation (2) with periodic velocity field

$$
u(x, t)=(2 x-1)^{2} \cos \{2 \pi t\}
$$

and source term

$$
\begin{gathered}
F(x, t)=-200 \cos \{2 \pi t\}(2 x-1+\sin \{2 \pi t\})\left(\pi+(2 x-1)^{2}\right) \\
\times \exp \left\{-50(2 x-1+\sin \{2 \pi t\})^{2}\right\}
\end{gathered}
$$

was used.

The initial condition was given by (16) with $t=0$ on $0 \leq x \leq 1$, and the boundary conditions were given by (16) with $x=0$ and $x=1$ for $0<t \leq T$.

The numerical solution was found using double precision in Fortran 90 on a Silicon Graphics Power Challenge, for various values of the grid-number $J$ and final time $T$. The time step was chosen to be

$$
\Delta t=|c|_{\max } / J
$$

Testing First Stability Criterion: "frozen" $u$ values With grid numbers $J$ in the range 100 to 500, which covers those used in most applications, the largest values of $|c|_{\max }$ for which each of the FDE's (3-6) remained stable 
for various values of $T$ up to 128 periods were determined. Some typical results for $|c|_{\max }$ are shown in Table 1 . Note that no sign of instability was detected in tests for values of $|c|_{\max } \leq 1$, even for $T$ up to 1024 periods and $J$ up to 10000 . Also, note that the results in the table did not change in the range 32 to 128 .

TABLE 1: Largest values of $|c|_{\max }$ for which FDE's (3-6) remain stable for 32 to 128 periods for grid numbers $J=100,300,500$, for Test Problem 1.

\begin{tabular}{|c||c|c|c|c|}
\hline Grid no. J & Eqn. (3) & Eqn. (4) & Eqn. (5) & Eqn. (6) \\
\hline \hline 100 & 1.58 & 1.63 & 1.63 & 1.61 \\
\hline 300 & 1.50 & 1.50 & 1.50 & 1.51 \\
\hline 500 & 1.48 & 1.43 & 1.49 & 1.47 \\
\hline
\end{tabular}

In a particular test, for a given value of $J$ and $|c|_{\max }$, instability was detected by examining the RMS errors at the last time step for each period. A sudden large rise in the slowly increasing RMS error at the end of successive cycles, say, from $6.89 \times 10^{-4}$ to $6.93 \times 10^{-4}$ to $6.96 \times 10^{-4}$ to $1.26 \times 10^{5}$, indicated that the result at the end of the second to last cycle showed no evidence of instability, whereas the RMS error at the end of the last cycle indicated that the result was affected by instability.

Table 1 shows that for $J$ up to 500 the largest value of $|c|_{\max }$ that can be used to give stable results did not change from $T=32$ to $T=128$, and all four FDE's were stable if $|c|_{\max } \leq 1.43$.

A partial explanation of the reason for stability when $|c|_{\max }>1$ is seen 


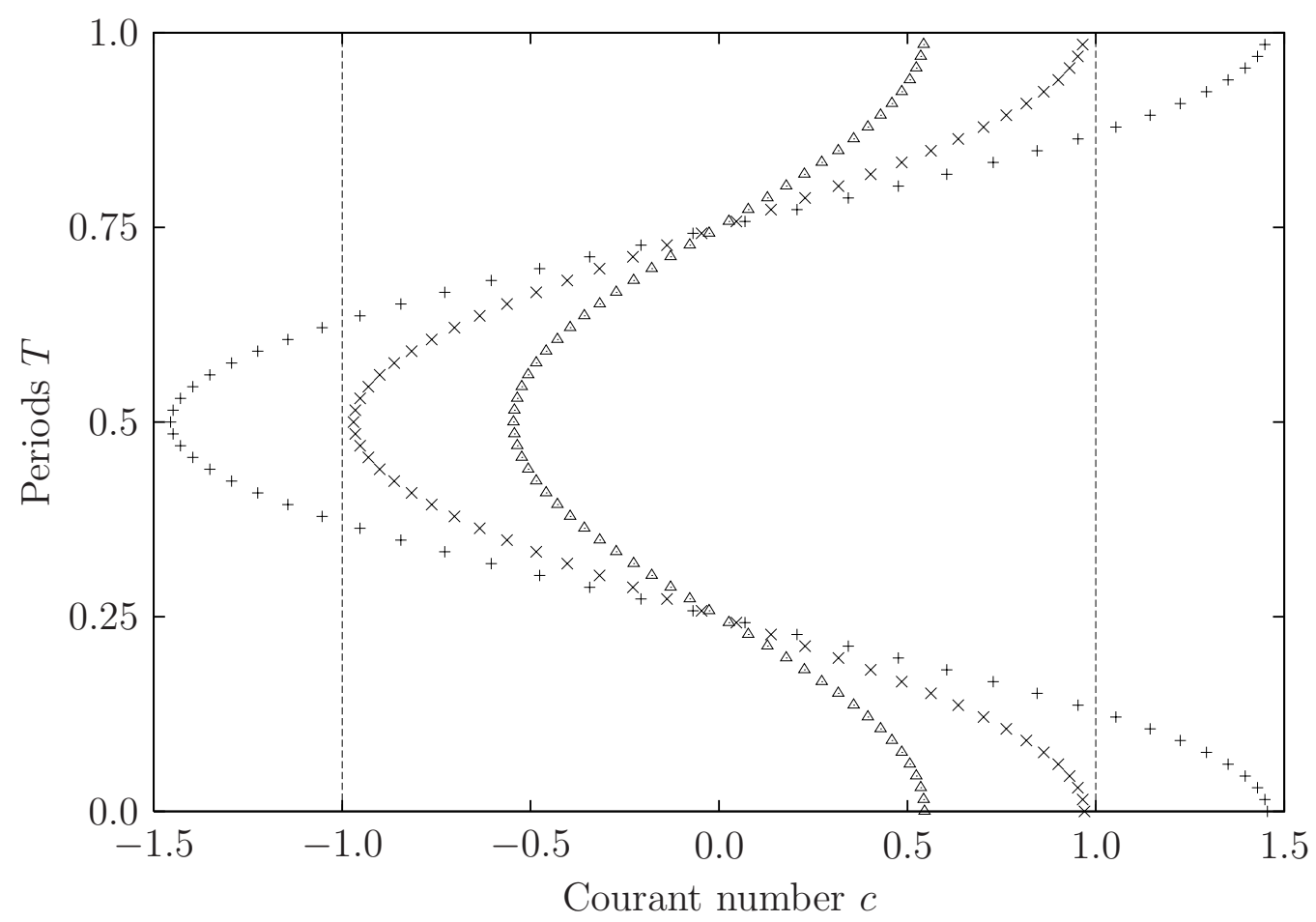

Figure 2: Values of the Courant number $c$ shown at each time step over one period with $|c|_{\max }=1.5$ and $J=100$, at $x=0.01$ shown,$+ x=0.1$ shown $\times$ and $x=0.2$ shown $\triangle$ 
in Figure 2. For $|c|_{\max }=1.5$, with $J=100$, at the point $x=0.01, x=0.1$ and $x=0.2$ the local Courant number used in the FDE's at each time step during one period are shown. The choice of $x=0.01$ produced the largest values of $|c|$ for all grid points between $0<x<1$.

At $x=0.01$, during one period 32 local values of $|c|$ are less than one and 34 are greater than one; at $x=0.1$ and $x=0.2$ all local values of $c$ are less than one during one period. Therefore, as the maximum velocity decreases from $x=0.01$ to $x=0.5$, then increases correspondingly, it is to be expected that most of the local applications of the four FDE's will occur with $|c|<1$. This is clearly seen for $x=0.1$ and $x=0.2$, for which $|c|<1$ for every time step in the entire period. In fact, this is true for every grid point in the range $0.1 \leq x_{j} \leq 0.9$. Since $|c|<1$ during most applications of the FDE's the corresponding von Neumann factor $\left|G_{j}^{n}\right|$ at the point $\left(x_{j}, t_{n}\right)$ (see Appendix A) is less than one, when round-off errors are reduced, for most applications. Note that, in Table 1 , for $J=100$ the value of $|c|_{\max }$ can be as large as 1.58 before instability might occur in the first 128 cycles.

Testing Second Stability Criterion: "frozen" $c$ and $d$ values In Figure 1 , superimposed on the stability region of the $(c, d)$ plane for FDE's $(4-6)$ is the set of points $(c, d)$ used during one period at the point $x=0.01$ when $J=100$ and $|c|_{\max }=1$, for this test problem. Clearly, for the upwind modification of Crowley's formula (Figure 1(a)) the local values of $c$ and $d$ lie outside the stability region, obtained using the "frozen" coefficients $c$ and $d$, about one-quarter of the time. From Figure 1(b), it is seen that the set of 
points $(c, d)$ lie outside the stability region for more than half the time for the centred-space modification of Crowley's formula. From Figure 1(c), the points $(c, d)$ lie within the stability region all the time.

Figure 3(a) shows the effect of increasing $J$ on the set of $(c, d)$ values obtained at $x=0.01$ for $|c|_{\max }=1.0$. According to this, one would expect stability to increase as $J$ increases, as more of the $(c, d)$ points lie in the stability region. However, tests have shown that increasing the values of $J$ for a given $|c|_{\max }>1$, cause instability if $J$ is large enough. For example (5) is stable up to $J=570$ when $|c|_{\max }=1.5$, and is unstable for $J>570$.

The number of $(c, d)$ points outside the "frozen" coefficients local stability region for the UW modification of Crowley's formula is 45 for $J=100$ in a total of 66 ; for the CS modification it is 51 and for the semi-Lagrangian FDE it is 36. Extrapolating these figures to other values of $J$ and other points $x$, it could be expected that (6) should be more stable than (4) followed by (5). In practice (see Tables 1 and 2) the stabilities for $J=100$ appear to be best for (4) and (5), followed by (6); but all are stable at $|c|_{\max }=1.5$.

\subsection{Test Problem 2: Spatial periodicity of $2 \Delta x$}

In an FDE the $2 \Delta x$ modes in the error distribution are the most sensitive to instability. It was therefore decided to test the stability of the FDE's (3-6) 

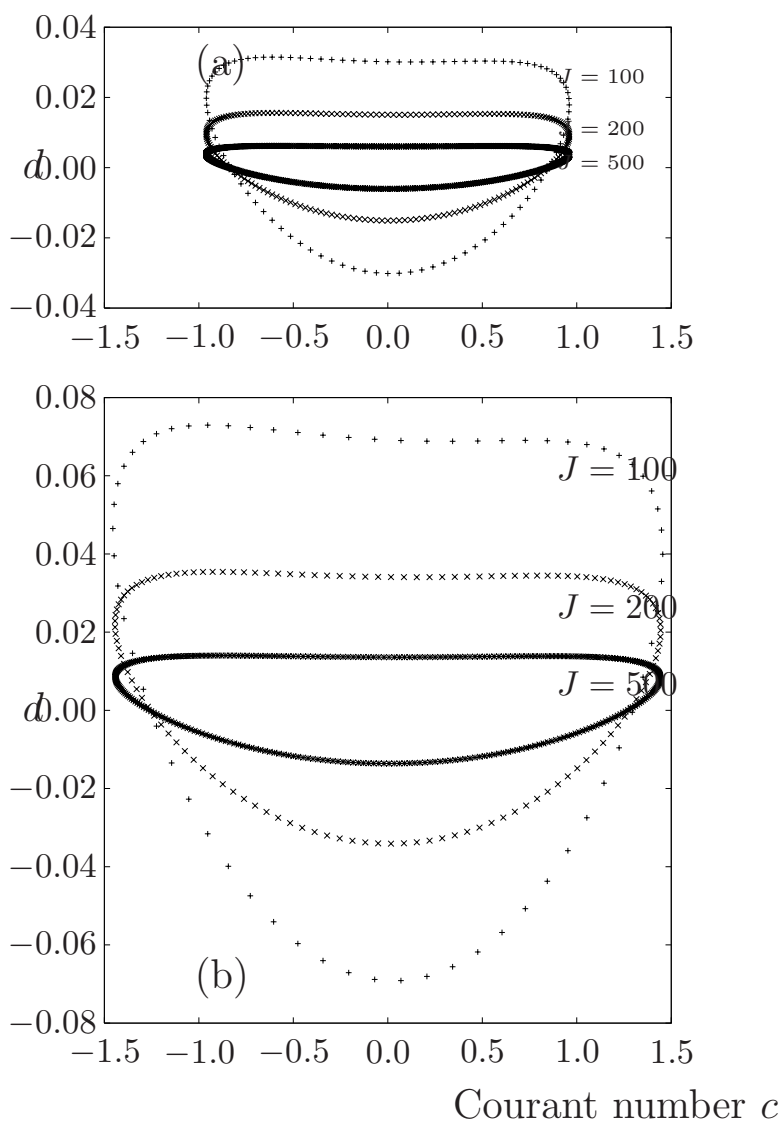

Figure 3: Values of $(c, d)$ shown for the point $x=0.01$ for $J=100,200,500$ for each time step, (a) for $|c|_{\max }=1.0$ and (b) for $|c|_{\max }=1.5$. 
with the variable velocity given by (17) and the source term given by

$$
\begin{gathered}
F(x, t)=-\pi\left[2 \sin (2 \pi t)+J(2 x-1)^{2} \sin (J \pi x) \cos (2 \pi t)\right] \\
\times \exp \{\cos (J \pi x)+\cos (2 \pi t)-2\}
\end{gathered}
$$

The exact solution to (2) in this case is

$$
\hat{\tau}(x, t)=\exp \{\cos (J \pi x)+\cos (2 \pi t)-2\}
$$

which has spatial periodicity of $2 / J=2 \Delta x$.

For this problem the initial condition is given by substituting $t=0$ in (21) and the boundary conditions are given by (21) with $x=0$ and $x=1$. The function (21) is oscillatory in space with $2 \Delta x$ periodicity.

TABLE 2: Largest values of $|c|_{\max }$ for which FDE's (3-6) remain stable for 32 to 128 periods for grid numbers $J=100,300,500$, for Test Problem 2.

\begin{tabular}{|c||c|c|c|c|}
\hline Grid no. $J$ & Eqn. (3) & Eqn. (4) & Eqn. (5) & Eqn. (6) \\
\hline \hline 100 & 1.58 & 1.63 & 1.63 & 1.61 \\
\hline 300 & 1.50 & 1.50 & 1.50 & 1.50 \\
\hline 500 & 1.47 & 1.38 & 1.48 & 1.47 \\
\hline
\end{tabular}

Similar results to those shown in Table 1 for Test Problem 1 were obtained for this test (see Table 2), indicating that the presence of $2 \Delta x$ modes in the error distribution for the first test problem behaved the same as in Test Problem 2. 


\section{Conclusion}

Numerical tests have shown that three recently developed second-order explicit FDE's for oscillatory advection with a source appear to be always stable if $|c|_{\max }$ is less than one. This is the condition obtained using Strikwerda's [10] stability criterion. The alternate method, that of using "frozen" coefficients in the finite-difference equation, is more difficult to apply and appears to be more restrictive requiring constraints in addition to $|c|_{\max } \leq 1$.

Investigations carried out with various practical values of the spatial grid number $J$ showed that the FDE's were also stable for some values of $|c|_{\max }$ greater than one; in fact, for $J$ up to 500 , all three new formulae as well as Crowley's standard formula, are stable when run up to 128 periods of the initial condition so long as $|c|_{\max } \leq 1.43$.

\section{A Appendix}

Substitution of $\tau_{j}^{n}=G^{n} \exp \{i \beta j\}$ into modification (6), in which $G$ is the von Neumann factor, $\beta$ is a wave number and $i=\sqrt{-1}$, yields

$$
G=1+c^{2}(\cos \beta-1)-i(c+d) \sin \beta .
$$

For stability, $|G| \leq 1$ is required for all $\beta$, giving the condition

$$
c^{4}(\cos \beta-1)^{2}+2 c^{2}(\cos \beta-1)+(c+d)^{2} \sin ^{2} \beta \leq 0 .
$$


Defining $\chi=\sin ^{2}\{\beta / 2\}$, when $\cos \beta=1-2 \chi$ and $\sin \beta=4 \chi(1-\chi)$, the requirement becomes

$$
F(\chi)=\left(c^{4}-(c+d)^{2}\right) \chi^{2}-\left(c^{2}-(c+d)^{2}\right) \chi \leq 0,
$$

for all $\chi$ in the range $0 \leq \chi \leq 1$. Since $F(\chi)$ is a quadratic with $F(0)=0$, this will be satisfied provided

$$
F^{\prime}(0)=d(d+2 c) \leq 0 \quad \text { and } \quad F(1)=c^{2}\left(c^{2}-1\right) \leq 0,
$$

both of which hold if $c^{2} \leq 1$ and either $d \geq-2 c$ if $d<0$ or $d<-2 c$ if $d \geq 0$.

\section{References}

[1] W. P. Crowley. Numerical advection experiments. Mon. Weath. Rev., 96:1-11, 1968. C1078, C1083

[2] P. D. Lax and R. D. Richtmyer. Survey of the stability of linear finite difference equations. Commun. Pure Appl. Math, 9:267-293, 1956. C1080

[3] C. E. Leith. Numerical Simulation of the Earth's Atmosphere. Technical Report 7986-T, UCRL, 1964. C1078, C1078, C1083

[4] A. R. Mitchell and D. F. Griffiths. The finite difference method in partial differential equations. John Wiley \& Sons, Chichester, 1980. C1079, C1084 
[5] K. W. Morton and D. F. Mayers. Numerical solution of partial differential equations. Cambridge University Press, Cambridge, 1996. C1084

[6] B. J. Noye. Some explicit three-level finite difference simulations of advection. Math. Comp. in Simul., 32:359-372, 1991. C1078

[7] B. J. Noye. Some three-level finite difference methods of simulating advection in fluids. Comput. Fluids, 19:119-140, 1991. C1078

[8] B. J. Noye. Explicit finite difference methods for variable velocity advection in the presence of a source. Comput. Fluids, 29(4):385-399, 2000. C1078, C1078, C1079, C1081, C1081, C1081, C1084

[9] G. G. O'Brien, M. A. Hyman, and S. A. Kaplan. A study of the numerical solutions of partial differential equations. J. Math. Phys., 29:23-251, 1950. C1079, C1083

[10] J. C. Strikwerda. Finite difference schemes and partial differential equations. Wadsworth \& Brooks/Cole, California, 1989. C1079, C1083, C1094 